\title{
Medical Service Competition among Heterogeneous Hospitals and Optimal Hybrid Medical Fee
}

\author{
Isao Miura \\ Kyushu University \\ Faculty of Economics \\ Japan
}

\begin{abstract}
This paper formulates the situation that a large hospital and a small hospital compete for the quality of chronic medical services using Hotelling's model and examine whether efficiency of chronic medical service and minimization of public health expenditure can be achieved simultaneously designing different hybrid chronic medical fee systems for each hospital. The main results were derived as follows. First, if both hospitals behave selfishly, then the abovementioned conditions can be achieved. Second, if the large hospital acts altruistically, then it selects excessive medical service whereas the small one provides inadequate levels. Third, in the case in which there is a brand effect for large hospital, I show by numerical example the possibility that the abovementioned conditions can be achieved by making the total reward of the large hospital greater than that of the small hospital. However, the number of patients for each hospital is not optimal.
\end{abstract}

Keywords: Medical Service Competition, Heterogeneous Hospitals, Hybrid Medical Fee, Game Theory JEL classification:L21, I11, I18

\section{Introduction}

Under circumstances in countries such as Japan, with free access permitted to patients, who are consumers of medical services and where freedom of opening the hospital is guaranteed to hospitals as the suppliers, competition among hospitals has become particularly intense in urban areas. Competition among hospitals can take various forms among general hospitals, among general hospitals, among special hospitals, among general hospitals and clinics, and even among clinics. Competition of many kinds is expected to intensify among hospitals offering similar services, but when medical service prices are regulated under the public medical insurance system, competition on medical service quality engenders improved benefits for the patient side. If medical fees are prescribed by the payment system, then the possibility exists that excessive medical services will be provided through competition. As a result, there occurs a problem by which public sector medical expenditures increase. In 2003, for hospitals of a certain size, DPC (diagnostic group classification comprehensive evaluation) systems were introduced in Japan. It is therefore expected that thesupply of excessive medical services will be suppressed ${ }^{1}$. However, even at hospitals to which DPC is applied, the payment for medical services for outpatients is used as the usual fee payment. Medical services for such outpatients are also being offered at small hospitals, which put them in mutual competition.

As described herein, I present discussion of competition related to chronic medical service quality of between large hospitals and small hospitals using Hotelling's spatial competition model. In general, situations in which firms with different objectives compete in terms of quantity and price in an oligopolistic market are analyzed as mixed oligopoly markets. Numerous research results have been obtained from analyses of such markets. Nevertheless, studies that have examined medical services as a mixed oligopoly market as described by PitaBarros and Martinez-Giralt (2002), Sanjo (2009), Miura and Maeda (2014), and others, are few. Those studies examine situations in which public hospitals and private hospitals are located at both ends of Hotellingtype linear city. Pita-Barros and Martinez-Giralt (2002) considered a case in which public hospitals and private hospitals compete in terms of quality and price of medical services.

\footnotetext{
${ }^{1}$ As another policy of restraining public health expenditure, the banning of mixed medical care and promotion of collaboration between medical care and nursing care are discussed in Japan. The former was considered by Miura(2014). The latter was examined by Miura and Tajika (2015).
} 
Then they assumed that public hospitals behave as first players whereas private hospitals behave as late players. They clarified the conditions under which medical services can be provided efficiently.By contrast, Sanjo (2009) and Miura and Maeda (2014), who specifically examined the Japan medical system, examined circumstances under which public hospitals and private hospitals mutually compete in terms of quality of medical services when the price of medical services is regulated. Sanjo (2009) assumed some uncertainty in the quality of medical services and demonstrated that public hospitals increase the quality of medical services when patients appreciate the quality of medical services. However, as described by Sanjo (2009), a patient's utility is defined linearly with respect to the quality of medical services. The quality of first-best medical services cannot be derived. Miura and Maeda (2014) define a patient's utility nonlinearly with respect to the quality of medical services to endogenize the quality of first-best medical services. They consider circumstances under which there is no uncertainty in the quality of medical services and where a public hospital chooses the quality of medical services to maximize social welfare. A private hospital chooses the quality of medical services to maximize profits. Generally, public hospitals have higher medical service quality. For that reason, patients are likely to use them excessively. Contrary to such a conjecture, Miura and Maeda (2014) demonstrated that efficient medical services can be provided in an environment with comprehensive medical fees. However these studies lack a public sector viewpoint of minimizing medical expenditures.

Miura (2016) deals not only with differences in objective functions of public or private hospitals as described above, but also with a situation in which each hospital has a different scale, a large hospital has an acute medical department and a chronic medical department, and each department decides the quality of medical services independently. By contrast, a small hospital consists only of a chronic medical department, which selfishly decides the quality of medical services. Considering such a framework, Miura (2016) designed a new medical fee compensation system that comprises fee payment and fixed payment ${ }^{2}$. Its main result is that when maintaining first-best medical services, public health expenditures can be reduced by lowering fixed fees and increasing variable compensation. As described in this paper, I further characterize differences between large hospitals and small hospitals: A large hospital has brand or reputation effects, but a small hospital has none. Subsequently, I investigate whether efficiency of chronic medical service and minimization of public health expenditure can be achieved simultaneously using different hybrid chronic medical fee systems for hospitals of each type. The main results were derived as follows. First, if not only the small hospital but also the large hospital behaves selfishly, then the efficiency of chronic medical service and minimization of public health expenditures can be achieved simultaneously. Second, if a large hospital acts altruistically, then the large hospital selects excessive medical services whereas the small hospital provides inadequate medical service. Third, in the case in which there is a brand effect for large hospitals, I show by numerical example the possibility that both efficiency of medical services and public health expenditure minimization can be achieved simultaneously by making the total reward of the large hospital greater than that of the small hospital. Then the number of patients attending the large hospital is too small compared with first best levels, whereas those of the small hospital become excessive.

The structure of this paper is the following. The following section formulates a basic model. I derive optimal quality of acute medical service for the large hospital. Section 3 examines quality of chronic medical services in both homogeneous competition and heterogeneous competition between a large hospital and a small hospital. Furthermore, I examine brand effects in large hospitals. The final section presents a summary of this paper and future tasks for related research.

\section{The Model}

We consider a Hotelling type of linear city in a closed interval $[0,1]$ in which small hospital and large hospital locate at extremes of the interval. The large hospital provides both a chronic medical service $v_{1}$ and an acute medical service $w$. However, the small hospital provides only chronic medical service $v_{0}$. Chronic disease patients are assumed to be uniformly distributed along the line of the interval. They can choose the hospital at which to have a medical examination. They have free access. Moreover it is assumed that each chronic patient will contract acute disease with probability $p$. The cost of chronic medical service $v_{i}(i=0,1)$ including chronic medical department of the large hospital is given as $\alpha v_{i}^{2} / 2$, where $\alpha$ is a positive parameter. Each patient bears the transportation cost $t$ per unit.

\footnotetext{
${ }^{2}$ Such a hybrid type of medical payment system is also used by Ma (1994), but his model incorporated only one medical institution. 
For these analyses, I assume that there is neither uncertainty nor asymmetry of information related to chronic medical services $v_{i}$ and acute medical services $w$. Moreover, I assume that both chronic medical services and acute medical services are provided under public medical insurance. Here, let $R\left(v_{i}\right)$ denote the medical fee per patient and lets $\in(0,1)$ denote the ratio of patient's self-payment for medical fee. Then one can represent the utility for patient located at point $x$ as

$$
\begin{aligned}
& u_{x}^{0}=A v_{0}-s R\left(v_{0}\right)-t x, \quad \text { (Acaseofgoingtothesmallhospital) } \\
& u_{x}^{1}=A v_{1}-s R\left(v_{1}\right)-t(1-x), \quad \text { (Acaseofgoingtothelargehospital) }
\end{aligned}
$$

where $A$ is positive parameter. Here I make the following assumption.

Assumption $1 A v-s R(v)$ increases in $v$.

This assumption implies that a certain level of chronic medical service is guaranteed. Consequently, each patient gets positive utility no matter which hospital is visited. Because a patient chooses a hospital offering higher utility, by Assumption 1 the number of patients in small hospital is $x$, which satisfies $u_{x}^{0}=u_{x}^{1}$. That is

$$
x=\frac{1}{2}+\frac{A\left(v_{0}-v_{1}\right)-s\left(R\left(v_{0}\right)-R\left(v_{1}\right)\right)}{2 t} .
$$

As shown in the equation above, I impose an additional condition for competition between the small hospital and the large hospital to take place.

Competitive condition $\left|A\left(v_{0}-v_{1}\right)-s\left(R\left(v_{0}\right)-R\left(v_{1}\right)\right)\right|<t$

Considering Assumption 1, this condition requires that there is not much difference in the quality of chronic medical services between hospitals. Consequently, $0<x<1$ if the Competitive condition holds. In the following section, the Competition condition is not so important in Section 3.1 that the chronic medical service cost and the norm of action are the same between hospitals because the equilibrium quality of chronic medical services is identical. However, I emphasize that the Competition condition plays an important role in Section 3.2 by which the norms of action differ between hospitals. Now the profit of each hospital is the following:

$$
\begin{gathered}
\pi_{0}=R\left(v_{0}\right)\left(\frac{1}{2}+\frac{A\left(v_{0}-v_{1}\right)-s\left(R\left(v_{0}\right)-R\left(v_{1}\right)\right)}{2 t}\right)-\frac{\alpha v_{0}^{2}}{2}, \\
\pi_{1}=R\left(v_{1}\right)\left(\frac{1}{2}+\frac{A\left(v_{1}-v_{0}\right)-s\left(R\left(v_{1}\right)-R\left(v_{0}\right)\right)}{2 t}\right)-\frac{\alpha v_{1}^{2}}{2} .
\end{gathered}
$$

Next, medical services for acute patients are explained. In this model, each acute patient receives medical service $w$ that the large hospital provides. The expected utility of the acute patient of spot $x$ is

$$
p(B w-s N-t(1-x))
$$

where $B$ is a positive parameter and $N$ represents the comprehensive medical fee for acute medical service. Then the large hospital's profitПobtained from acute medical service is calculable as follows:

$$
\Pi=p N-\frac{\beta w^{2}}{2},
$$

where $\beta w^{2} / 2$ denotes cost function for acute medical service $w$ and $\beta$ is positive constant. I assume that the large hospital chooses the acute medical service $w$ altruistically considering not only the profit obtained from acute patients but also the patient's utility to some degree. Then objective function $H$ for the large hospital is defined as

$H=p\left(N+\theta \int_{0}^{1}\left(B w-s N-t\left(1-x^{\prime}\right)\right) d x^{\prime}\right)-\frac{\beta w^{2}}{2}$

where $\theta(\in(0,1))$ represents a weight parameter on patient's utility. Acute medical service $w$ which maximizes $H$ is obtainable from the first-order condition $d H / d w=0$ as

$$
w=\frac{p \theta B}{\beta}(9)
$$

The equation above implies that the greater the weight parameterOor the onset probability $p$ becomes, the better the quality of acute medical service $w$ becomes. Especially when $\theta=1$, the first acute best medical servicew $=$ $(p B) / \beta$ is obtainable. 


\section{Determination of chronic medical services}

Now specifying the medical fee per patient $R\left(v_{i}\right)$ as linear scheme $a v_{i}+b(a \geq 0, b \text { : constant })^{3}$, I derive chronic medical service in competition between small hospital and a large hospital.

\subsection{Homogeneous competition}

Next, one can examine the case in which the large hospital determines the level of chronic medical service considering only the hospital's own profit. Then the objective function for the large hospital is

$$
\pi_{1}=\left(a v_{1}+b\right)\left(\frac{1}{2}+\frac{(A-s a)\left(v_{1}-v_{0}\right)}{2 t}\right)-\frac{\alpha v_{1}^{2}}{2},
$$

and the profit of the small hospital is

$$
\pi_{0}=\left(a v_{0}+b\right)\left(\frac{1}{2}+\frac{(A-s a)\left(v_{0}-v_{1}\right)}{2 t}\right)-\frac{\alpha v_{0}^{2}}{2} .
$$

Here I make an additional assumption.

Assumption $23 A^{2}-8 s t \alpha<0$.

Because Assumption 2 implies $2 t \alpha>3 a(A-s a)$ for any $a$, it ensures the uniqueness and stability of Nash equilibrium chronic medical service under homogeneous competition ${ }^{4}$. Moreover by Assumption 2 , the secondorder condition of profit maximization for each hospital, $\partial^{2} \pi_{i} / \partial v_{i}^{2}=a(A-s a) / t-\alpha<0$ holds. Then by the first-order condition $\partial \pi_{i} / \partial v_{i}=0(i=0,1)$, the reaction functions for each hospital are the following:

$$
\begin{gathered}
v_{1}=\frac{a(A-s a) v_{0}-a t-b(A-s a)}{2[a(A-s a)-t \alpha]}, \\
v_{0}=\frac{a(A-s a) v_{1}-a t-b(A-s a)}{2[a(A-s a)-t \alpha]} .
\end{gathered}
$$

By Assumptions 1 and 2, both the coefficient of $v_{0}$ on the right side in equation (12) and the coefficient of $v_{1}$ on the right side in equation (13) are negative when $a>0$. Consequently, medical services for chronic disease competition between a small hospital and a large hospital has a strategic substitutive relation under a volume fee payment system ${ }^{5}$. Under comprehensive payment system $(a=0)$, each hospital has an optimal strategy irrespective of the rival's strategy by equations (12) and (13). Because equations (12) and (13) are symmetric, it holds that $v_{0}=v_{1}$. Let $v_{*}$ denote the solution. Then $v_{*}$ is the following:

$$
v_{*}=\frac{a t+b(A-s a)}{2 t \alpha-a(A-s a)} \text {. }
$$

By the second-order condition of profit maximization for each hospital, the denominator of the right side in equation (14) is positive. Therefore, it holds that $v_{*}>0$. Here I examine comparativestatics related to the effect on $v_{*}$ of $a$ and $b$. As might be readily apparent from equation (14), $v_{*}$ improves as the fixed fee $b$ increases. The effect of $a$ is ambiguous. Next, the optimal medical fee is derived. Let $W$ denote social welfare for chronic medical service as

$$
W=a v_{*}+b-\alpha v_{*}^{2}+2 \int_{0}^{1 / 2}\left(A v_{*}-s\left(a v_{*}+b\right)-t x^{\prime}\right) d x^{\prime}-(1-s)\left(a v_{*}+b\right)
$$

where the last term $(1-s)\left(a v_{*}+b\right)$ of the right side in the equation above implies medicalexpenditures by public insurers (government). Calculating equation (15) gives

$$
W=a v_{*}-\alpha v_{*}^{2}-\frac{t}{4}
$$

\footnotetext{
${ }^{3}$ Especially when $a>0$ and $b=0$, it means volume fee payment system, when $a=0$ and $b>0$, it signifies comprehensive payment system.

${ }^{4}$ Assumption 2 implies the condition by which the discriminant of quadratic equation for, $3 \mathrm{~s} a^{2}-3 A a+2 t \alpha=0$, is negative; it holds that $2 t \alpha>3 a(A-s a)$. Actually $2 t \alpha>3 a(A-s a)$ represents the magnitude relation of the intercepts of the linear response functions for the large hospital and the small hospital that will be derived hereinafter.

${ }^{5} \mathrm{~A}$ case in which quality competition brings about strategic substitution is also obtained in Pita-Barros and Martinez-Giralt (2002). By contrast, Montefiori (2005) and Sanjo (2009) exhibit strategic complementary relations on quality competition using a comprehensive payment system and linear cost function on the quality of medical services per patient.
} 
Maximizing $W$ with respect to $v_{*}$, the optimal chronic medical service in the sense of first best isthe following:

$$
v_{*}=\frac{A}{2 \alpha} \text {. }
$$

Consequently, to reconcile Nash equilibrium $v_{*}$ with equation (17), $a$ and $b$ must satisfy the following relation:

$$
b=\frac{t A(s-1)}{s(A-s a)}+\frac{t}{s}-\frac{a A}{2 \alpha} .
$$

Here, it is noteworthy that social welfare $W$ is independent of medical fee $a v_{*}+b$ by equation (16).Therefore, to solve $a$ and $b$ completely, other conditions are necessary for them. New conditions for minimizing public health expenditure $a v_{*}+b$ are introduced. The condition is formalized as guaranteed non-negative profit for each hospital $^{6}$. Therefore because $a v_{*}+b-\alpha v_{*}^{2}=0$,

$$
b=\frac{A^{2}}{4 \alpha}-\frac{a A}{2 \alpha}
$$

By equations (18) and (19), I obtain the following proposition.

Proposition 1 An optimal medical fee that maximizes social welfare and minimizes public health expenditure is

$$
a=\frac{4 t \alpha A-A^{3}}{4 t \alpha-s A^{2}}, \quad b=\frac{A^{2}\left((2-s) A^{2}-4 t \alpha\right)}{4 \alpha\left(4 t \alpha-s A^{2}\right)}-\frac{a A}{2 \alpha} .
$$

Actually, $a$ in Proposition 1 is positive when $4 t \alpha>A^{2}$ and $b$ generally will not become 0 . Consequently, I assert that optimal medical fees are characterized as the compensation scheme using both the volume fee payment and comprehensive payment.

\subsection{Heterogeneous competition}

This subsection presents an examination of a situation in which a large hospital altruistically chooses the level of chronic medical service as a selection method of acute medical service. Then the large hospital's objective function $L$ for chronic medical service is definable as

$$
L=(1-x)\left(a v_{1}+b\right)-\frac{\alpha v_{1}^{2}}{2}+\theta \int_{0}^{1 / 2}\left(A v_{1}-s\left(a v_{1}+b\right)-t\left(1-x^{\prime}\right)\right) d x^{\prime}
$$

where $x$ satisfies

$$
x=\frac{1}{2}+\frac{(A-s a)\left(v_{0}-v_{1}\right)}{2 t}
$$

Substituting equation (21) into equation (20) and by arranging terms, $L$ can be written as

$$
\begin{aligned}
& L=\left(a v_{1}+b\right)\left(\frac{1}{2}+\frac{(A-s a)\left(v_{0}-v_{1}\right)}{2 t}\right)-\frac{\alpha v_{1}^{2}}{2} \\
& +\theta\left[A v_{1}-s\left(a v_{1}+b\right)-t\right]\left(\frac{1}{2}+\frac{(A-s a)\left(v_{1}-v_{0}\right)}{2 t}\right) \\
& +\frac{t \theta}{2}\left[1-\left(\frac{1}{2}+\frac{(A-s a)\left(v_{0}-v_{1}\right)}{2 t}\right)^{2}\right] .
\end{aligned}
$$

To derive $v_{1}$ that maximizes $L$, one can partially differentiate $L$ with respect to $v_{1}$ :

$$
\begin{aligned}
\frac{\partial L}{\partial v_{1}}= & \frac{(A-s a)\left(a v_{1}+b\right)}{2 t}+\frac{a(A-s a)\left(v_{1}-v_{0}\right)}{2 t}+\frac{a}{2}-\alpha v_{1} \\
& +\frac{\theta(A-s a)}{2 t}\left[A v_{1}-s\left(a v_{1}+b\right)-t\right] .
\end{aligned}
$$

\footnotetext{
${ }^{6}$ Because the public sector designs the medical fees for acute medical service and chronic medical service separately, the profit of the large hospital is reflected in the chronic medical service department.
} 
The second line in equation (23) is positive by Assumption 1 and Competitive condition ${ }^{7}$. Therefore, in this case, a reaction curve for large hospital makes a shift upward. However, the reaction curve for small hospital remains unchanged as a homogeneous case in the previous subsection. From the discussion presented above, if $a, b$ by the given Proposition 1 as medical fee ${ }^{8}$, then the following proposition can be obtained:

Proposition 2 Under heterogeneous competition the chronic medical service $v_{1}$ is excessive. Also, $v_{0}$ is too small compared with each first best level.

Consequently, the number of patients at the large hospital will be larger than that of the small hospital. Hence inefficiency will occur. This result supports outcomes obtained in the earlier mixed oligopoly market literature.

\subsection{Brand effect at the large hospital}

This subsection presents consideration of a situation in which chronic disease patients obtain additional benefit $V\left(\in\left(0, t-A^{2} / \alpha\right)\right)$ only when visiting a large hospital. This benefit represents a brand effect that is unique to a large hospital backed by medical technology that is capable of providing acute care. In a small hospital, such a brand effect does not occur as before. Then it would be justifiable to use different treatment fee schemes for a large hospital and a small hospital.For medical services for chronic disease $v_{i}(i=0,1), a_{i} v_{i}+b_{i}\left(a_{i}>0, i=\right.$ $0,1)$ is defined as a medical fee, where $a_{i}$ and $b_{i}$ are constant. Then the utility for patient located at point $x$ as

$$
\begin{aligned}
& u_{x}^{0}=A v_{0}-s\left(a_{0} v_{0}+b_{0}\right)-t x, \\
& u_{x}^{1}=A v_{1}-s\left(a_{1} v_{1}+b_{1}\right)-t(1-x)+V .
\end{aligned}
$$

Here I find the first best solution. In this case, social welfare $W$ can be written as

$$
\begin{gathered}
W=\left(a_{0} v_{0}+b_{0}\right) x-\frac{\alpha v_{0}^{2}}{2}+\left(a_{1} v_{1}+b_{1}\right)(1-x)-\frac{\alpha v_{1}^{2}}{2}+\int_{0}^{x}\left(A v_{0}-s\left(a_{0} v_{0}+b_{0}\right)-t x^{\prime}\right) d x^{\prime} \\
\quad+\int_{x}^{1}\left(A v_{1}-s\left(a_{1} v_{1}+b_{1}\right)-t\left(1-x^{\prime}\right)+V\right) d x^{\prime} \\
-(1-s)\left(a_{0} v_{0}+b_{0}\right) x-(1-s)\left(a_{1} v_{1}+b_{1}\right)(1-x) \\
=-\frac{\alpha v_{0}^{2}}{2}-\frac{\alpha v_{1}^{2}}{2}+\int_{0}^{x}\left(A v_{0}-t x^{\prime}\right) d x^{\prime}+\int_{x}^{1}\left(A v_{1}-t\left(1-x^{\prime}\right)+V\right) d x^{\prime}
\end{gathered}
$$

The first best solution $\left(x^{F B}, v_{0}^{F B}, v_{1}^{F B}\right)$ maximizing $W$ is calculable as

$$
\begin{gathered}
x^{F B}=0<\frac{t \alpha-A^{2}-\alpha V}{2\left(t \alpha-A^{2}\right)}<\frac{1}{2}, \\
v_{0}^{F B}=\frac{A\left(t \alpha-A^{2}-\alpha V\right)}{2\left(t \alpha-A^{2}\right)}, \\
v_{1}^{F B}=\frac{A\left(t \alpha-A^{2}+\alpha V\right)}{2\left(t \alpha-A^{2}\right)} .
\end{gathered}
$$

In the first best solution, the quality of the chronic medical service in the large hospital is higher thanthat in the small hospital. The number of patients is greater in the large hospital than in the smallhospital. Medical fee $\left(a_{0}, b_{0}, a_{1}, b_{1}\right)$ is derived, which minimizes the reimbursement expenditure ofthe public sector $(1-s)\left[x^{F B}\left(a_{0} v_{0}^{F B}+b_{0}\right)+\left(1-x^{F B}\right)\left(a_{1} v_{1}^{F B}+b_{1}\right)\right]$ and which realizes the first bestsolution on Nash equilibrium under free access. Under such a medical fee scheme, the participationcondition of each hospital becomes binding. From (24) and (25), the number of patients in the smallhospital under free access can be expressed as follows:

$$
x=\frac{1}{2}+\frac{A\left(v_{0}-v_{1}\right)-s\left(a_{1} v_{1}+b_{1}-a_{0} v_{0}-b_{0}\right)-V}{2 t} .
$$

\footnotetext{
${ }^{7}$ In this case Competitive condition might restrict the range of possible values of $\theta$. However, no difficulty arises if $\theta$ is a very small positive number.

${ }^{8}$ Both hospitals have identical cost functions for chronic medical services. Therefore, using medical fees can be justified under homogeneous competition. 
Then profits of each hospital can be written as shown below.

$$
\begin{gathered}
\pi_{1}=\left(a_{1} v_{1}+b_{1}\right)\left(\frac{1}{2}+\frac{A\left(v_{1}-v_{0}\right)-s\left(a_{1} v_{1}+b_{1}-a_{0} v_{0}-b_{0}\right)+V}{2 t}\right)-\frac{\alpha v_{1}^{2}}{2} \\
\pi_{0}=\left(a_{0} v_{0}+b_{0}\right)\left(\frac{1}{2}+\frac{A\left(v_{0}-v_{1}\right)-s\left(a_{0} v_{0}+b_{0}-a_{1} v_{1}-b_{1}\right)-V}{2 t}\right)-\frac{\alpha v_{0}^{2}}{2}
\end{gathered}
$$

The Nash equilibrium $\left(v_{0}, v_{1}\right)$ satisfies the following condition:

$$
\begin{aligned}
& \frac{\partial \pi_{1}}{\partial v_{1}}=a_{1}\left(\frac{1}{2}+\frac{A\left(v_{1}-v_{0}\right)-s\left(a_{1} v_{1}+b_{1}-a_{0} v_{0}-b_{0}\right)+V}{2 t}\right) \\
& +\left(a_{1} v_{1}+b_{1}\right) \frac{A-s a_{1}}{2 t}-\alpha v_{1}=0 \\
& \frac{\partial \pi_{0}}{\partial v_{0}}=a_{0}\left(\frac{1}{2}+\frac{A\left(v_{0}-v_{1}\right)-s\left(a_{0} v_{0}+b_{0}-a_{1} v_{1}-b_{1}\right)-V}{2 t}\right) \\
& +\left(a_{0} v_{0}+b_{0}\right) \frac{A-s a_{0}}{2 t}-\alpha v_{0}=0 .
\end{aligned}
$$

Substituting (28) and (29) into (31) (34) and letting (31) and (32) equal 0 , one can derive $\left(a_{0}, b_{0}, a_{1}, b_{1}\right)$ that satisfies the desired medical fee scheme. However, even with such a medical fee scheme, the number of patients at each hospital under free access is not optimal.

Proposition 3 When there exists a brand effect by visiting the large hospital, the medical fee scheme that realizes a first-best medical service for each hospital on the Nash equilibrium under free access and that minimizes the total medical fee does not optimize the number of patients at each hospital.

The proof of Proposition 3 will be done by raising a counter example with the following numerical example.

$$
A=t=1, V=0.1, \alpha=2, s=0.5
$$

Substituting these numerical values into (27) $\sim(29)$, one obtains $x^{F B}=0.4, v_{0}^{F B}=0.2, v_{1}^{F B}=0.3$.

By substituting these first best values and $R_{0}=a_{0} v_{0}+b_{0}, R_{1}=a_{1} v_{1}+b_{1}$ into (31) and (32) and by letting (31) and (32) equalize 0 , the following equations are obtained:

$$
\begin{gathered}
25 R_{1}^{2}-\left(60+25 R_{0}\right) R_{1}+9=0, \\
25 R_{0}^{2}-\left(40+25 R_{1}\right) R_{0}+4=0,
\end{gathered}
$$

Approximate solutions of (35) and (36) are $^{9}$

$$
R_{1}=0.153=0.3 a_{1}+b_{1}, \quad R_{0}=0.0968=0.2 a_{0}+b_{0} .
$$

The medical fee increases at the large hospital. This result is similar to the role of the Pigouvian subsidy policy on positive external effects. From (33), (34), and (37), one can obtain the following

$$
a_{1}=0.956, b_{1}=-0.1368, a_{0}=0.9036, b_{0}=-0.08372 \text {. }
$$

The fixed medical fee is negative for both hospitals. The variable one is greater for the large hospital. By substituting each numerical value into (30),

$$
x=0.41325>x^{F B} .
$$

Under free access, the number of patients at the small hospital is excessive. The number of the large hospital is too small. Therefore, to ascertain the number of first-best patients at each hospital, access control by the public sector might be necessary.

\section{Concluding Remarks}

As described in this paper, I have examined a case in which a large hospital and a small hospital compete for chronic medical services under free access in a Hotelling type linear city using a hybrid medical fee system that has a linearly combined payment compensation fee and a fixed payment fee. Specifically, I theoretically examined how the chronic medical service provided at each hospital will be affected when the action principle of a large hospital is selfish and altruistic, or when the large hospital has a brand effect. The main results are presented below. First, I demonstrated that competition under free access produced a first-best chronic medical service if the behavioral principles of a large hospital and a small hospital are common (both selfish).

\footnotetext{
9 'Mathematica' Software was used for its calculation.
} 
Second, when the large hospital acts altruistically, a large hospital will have excessive chronic medical services, whereas the small hospital will be at an underestimated chronic medical service level. Third, when a brand effect is applicable for consultation at a large hospital, although it is possible to provide first-best chronic medical services at both hospitals, numerical examples show that the number of consultations at large hospitals is too small, although the number of examinees at small hospitals is excessive.

Some attention must be devoted to the results presented above, ignoring that the increase in the number of consultations at hospitals lengthens waiting times. Particularly, when a waiting time occurs at a large hospital, the quality of chronic medical service is reduced. It might offset the brand effect. To alleviate congestion, it will be necessary to consider regulation of patient access. Because the third result depends only on numerical examples, it is apparently necessary to examine brand effects of large hospitals in a more general framework. Moreover, by expanding the analysis of this paper, one can consider cases with one large hospital and multiple small hospitals, with competition not only between large hospitals and small hospitals, but also between small hospitals, which cannot be modeled properly using a linear urban space model. Therefore, using a circular urban space model and designing situations with large hospitals in the center of the circle and small hospitals at the periphery, it is possible to analyze competition of both kinds simultaneously ${ }^{10}$. Related details will be examined in future studies.

\section{References}

Gravelle, H., 1999. “Capitation Contracts: Access and Quality,”Journal of Health Economics, 18, pp.315-340.

Pita-Barros, P., and X. Martinez-Giralt 2002."Public and Private Provision of Health Care," Journal of Economics \&Management Strategy, 3, pp.93-112.

Ma, A., 1994. "Health Care Payment systems: Cost and Quality Incentive," Journal of Economics \&Management Strategy, 11, pp.109-133.

Montefiori, M., 2005."Spatial competition for quality in the market for hosptal care,"European Journal of Health Economics, 6, pp.131-135.

Sanjo, Y., 2009. "Quality choice in a health care market: a mixed duopoly approach," European Journal of Health Economics, 10, pp.207-215.

Miura, I. and R. Maeda, 2014. "Shinryou Service no Shitsu ni Kansuru Kyousou to Shinryou Housyu Seido," Kyushu University Economic Society Keizaigaku Kenkyu, 81(4), pp.123-134. (in Japanese)

Miura, I., 2014. "Kongo Shinryo to Iryokikan no Kyouso,"Kyushu Keizaigakkai Nenpo 52,pp.167-174. (in Japanese)

Miura, I. and H. Tajika, 2015. "Iryou Kaigo Service no Renkei to Saiteki Houkastu Houshu," Faculty of Economics of Kyushu University, Discussion Paper No. 2015-3, pp.1-10. (in Japanese)

Miura, I., 2016."Kotonaru Kodokihan to Kino wo Yusuru Byouin Kan no Kyousou," Faculty of Economics in Kyushu University, Discussion Paper No. 2016-7, pp.1-9. (in Japanese)

Nuscheler, R., 2003."Physician Reimbursement, time Consistency, and the Quality of Care," Journal of Institutional and Theoretical Economics, 159, pp.302-322.

\footnotetext{
${ }^{10}$ Gravelle (1999) and Nuscheler (2003) can be regarded as a study that examines competition among hospitals using a circular model. However, in their analysis, the presence of a core hospital has been abandoned. 